\title{
Correlation of Unstimulated and Stimulated Salivary Flow Rate in Chronic Periodontitis
}

\author{
Mohammed Alasqah
}

\section{ABSTRACT}

Aim: The present study aimed at correlating the flow rate of the stimulated and unstimulated saliva in chronic periodontitis.

Materials and methods: This study composed of 100 subjects between the age group of 25 years and 45 years. These subjects are categorized into four groups, placing 25 subjects in each. Group 1 with 25 healthy patients (no loss of attachment clinically), group 2 with 25 mild chronic periodontitis subjects (1-2 mm of attachment level clinically), group 3 with 25 moderate chronic periodontitis subjects $(3-4 \mathrm{~mm}$ of attachment level clinically), group 4 with 25 severe chronic periodontitis subjects ( $5 \mathrm{~mm}$ of attachment level clinically), split method was used to collect the stimulated and unstimulated saliva. A calibrated digital balance was used to weigh the bottle before and after the collection of saliva. The result was considered to be statistically significant at a $p$ value less than $0.05(p<0.05)$.

Result: The stimulated and unstimulated flow rate of saliva was high in group 1 subjects $(0.920 \pm 0.240,1.366 \pm 0.280)$ followed by group 2 subjects $(0.780 \pm 0.246,0.920 \pm 0.146)$, group 3 subjects $(0.408 \pm 0.132,0.590 \pm 0.110)$, and group 4 subjects $(0.221 \pm 0.348,0.301 \pm 0.216)$. There was a significant difference in the salivary flow rate between the groups statistically with $p$ value 0.0001 .

Conclusion: The stimulated and unstimulated salivary flow rate decreased with the severity of the progression of the chronic periodontitis.

Clinical significance: The importance of saliva on oral function includes antimicrobial activity, buffering function of $\mathrm{pH}$, cleansing action, mastication, and swallowing. Therefore, a decrease in the ability to produce saliva leads to various oral infections.

Keywords: Chronic periodontitis, Pocket depth, Salivary flow rate, Spit method.

How to cite this article: Alasqah M. Correlation of Unstimulated and Stimulated Salivary Flow Rate in Chronic Periodontitis. World J Dent 2019;10(1):58-61.

Source of support: Nil

Conflict of interest: None

\section{IINTRODUCTION}

The periodontitis in human is a group of an inflammatory disorder. Loe defined periodontitis as "a group of lesions

Department of Preventive Dental Sciences, Division of Periodontics, College of Dentistry, Prince Sattam Bin Abdul Aziz University, Al Kharj, Riyadh, Kingdom of Saudi Arabia

Corresponding Author: Mohammed Alasqah, Department of Preventive Dental Sciences, Division of Periodontics, College of Dentistry, Prince Sattam Bin Abdul Aziz University, Al Kharj, Riyadh, Kingdom of Saudi Arabia, e-mail: mohasqah@gmail.com affecting the tissues surrounding and supporting the teeth in their sockets" characterized by gum and other teeth supporting structure's inflammation. ${ }^{1}$ Recently, periodontitis is known to be caused from host response and bacterial infection's complex interplay generally modified by behavioral factors. The bacterial activity can eliminate the host tissue further leading to loss of periodontal attachment, loss of bone and finally loss of tooth. ${ }^{2}$ The recent studies on influencing factors for the manifestation and progression of the disease showed that the bacterial supposed to be associated with the periodontal disease are only of little significance. ${ }^{3}$

The important oral functions of the saliva include antimicrobial activity, buffering function of $\mathrm{pH}$, cleansing action, mastication, and swallowing. ${ }^{4}$ Therefore, a decrease in the ability to produce saliva leads to various oral infections. Though, the actual information regarding the conditions associated with the production of the saliva is limited..$^{5}$

Using the salivary flow rate, saliva's quantitative state is determined. The salivary flow rate is the measurement of production of the saliva in a given period of time by the salivary gland which is expressed usually in grams per minute or milliliters per minute $(\mathrm{g} / \mathrm{min}$ or $\mathrm{mL} / \mathrm{min})$. The difference in the normal values of the salivary flow rate was noted from several studies. This variation can be due to variation in the age, race, sex, geographical area, and the genetic difference determined by different studies. ${ }^{6,7}$ Therefore, this study was conducted to correlate the stimulated and unstimulated salivary flow rate in the condition of chronic periodontitis.

\section{MATERIALS AND METHODS}

\section{Study Population}

This study included 100 subjects aged between 25 years and 45 years from the clinic, College of Dentistry, Prince Sattam Bin Abdul Aziz University, Al Kharj, Kingdom of Saudi Arabia. A comprehensive exam was conducted on subjects who signed the consent form. The subjects with at least 10 teeth were included in the study. The subjects who were not able to provide sufficient data, who had less than 10 teeth, who have previous history of periodontal treatment, who is under any medication at the time of study, any history of having systemic and/or autoimmune diseases such as hormonal imbalance, diabetes, rheumatoid 
arthritis, systemic lupus erythematosus and Sjogren's syndrome were not included from the study.

The subjects were divided into four groups, 25 in each group:

- Group 1: 25 healthy patients (no clinical attachment loss)

- Group 2: 25 mild chronic periodontitis patients (clinical attachment level of 1-2 mm)

- Group 3: 25 moderate chronic periodontitis patients (clinical attachment level of 3-4 $\mathrm{mm}$ )

- Group 4: 25 severe chronic periodontitis patients (clinical attachment level of $5 \mathrm{~mm}$ )

\section{Calibration of the Examiner}

The principal investigator was responsible for the collection of data. To know the intraexaminer difference, a kappa value was calculated (0.8), which was considered to be sufficient for the study. The examination was conducted on every 20th participant to check the error in the examination.

\section{Collection of Unstimulated and Stimulated Saliva}

The spit method was used to collect unstimulated saliva for 5 minutes. The saliva was collected during a specific time period between 8:30 am and 11:30 pm. using a milliliter per minute scale, the salivary flow rate was calculated. The saliva was calculated before conducting the clinical examination to avoid the unwanted stimulation of the minor and major salivary glands when examining with intraoral equipment. The subjects were informed clearly about the study protocol and usage of a stopwatch to check the saliva collection period. A plastic bottle of 40 $\mathrm{mL}$, with $5 \mathrm{~cm}$ in diameter and a tight-fitting lid, was used to collect saliva. The empty bottle before and after the saliva collection was weighed using a calibrated digital balance (JY-09, Twins electronic Kitchen scale) which is near to $0.1 \mathrm{mg}$. The weight difference was calculated after 5 minutes of the collection period. The weight was divided by the duration ( $5 \mathrm{~min}$ ) to find the salivatory flow rate of the individual.
Subjects were instructed to sit still and lean forward head over the plastic bottle. Subjects were instructed to chew the chewing gum following the pace of the metronome (approx. 70 strokes/min). For every minute, the subjects were instructed to spit the saliva in the bottle without swallowing.

\section{Clinical Assessment}

The periodontal attachment level was measured clinically using a conventional periodontal probe from the four points of the teeth. The probe was inserted parallelly along the long axis of the tooth. The pocket depth was measured in each tooth from the gingival margin to the base at six sites.

\section{RESULT}

The association of the chronic periodontitis with the unstimulated salivary flow rate is presented as Table 1. The flow rate was more in healthy subjects $(0.920 \pm 0.240)$, followed by subjects with mild chronic periodontitis $(0.780 \pm 0.246)$, subjects with moderate chronic periodontitis $(0.408 \pm 0.132)$ and subjects with severe chronic periodontitis $(0.221 \pm 0.348)$. There was a significant difference between the group statistically with a $p$ value less than 0.05 ( $p$ 0.0001).

The association of the chronic periodontitis with the stimulated salivary flow rate is presented as Table2. The flow rate was more in healthy subjects $(1.366 \pm 0.280)$, followed by subjects with mild chronic periodontitis $(0.920 \pm 0.146)$, subjects with moderate chronic periodontitis $(0.590 \pm 0.110)$ and subjects with severe chronic periodontitis $(0.301$ $\pm 0.216)$. There was a significant difference between the group statistically with a $p$ value less than 0.05 ( $p$ $-0.0001)$. The salivary (stimulated and unstimulated) flow rate of chronic periodontitis patients is represented in Graph 1.

\section{Statistical Analysis}

The analysis of data was done using SPSS software version 17.0 with a one-way analysis of variance (ANOVA).

Table 1: Association of unstimulated salivary flow rate with chronic periodontitis patients

\begin{tabular}{lllc}
\hline Groups & $N$ & Mean $\pm S D$ & F value \\
Group 1 & 25 & $0.920 \pm 0.240$ & \\
Group 2 & 25 & $0.780 \pm 0.246$ & 26.146 \\
Group 3 & 25 & $0.408 \pm 0.132$ & $0.0001^{*}$ \\
Group 4 & 25 & $0.221 \pm 0.348$ & \\
\hline
\end{tabular}

Table 2: Association of stimulated salivary flow rate with chronic periodontitis patients

\begin{tabular}{llll}
\hline Groups & $N$ & Mean $\pm S D$ & $F$ value \\
\hline Group 1 & 25 & $1.366 \pm 0.280$ & \\
Group 2 & 25 & $0.920 \pm 0.146$ & 29.649 \\
Group 3 & 25 & $0.590 \pm 0.110$ & $0.0001^{*}$ \\
Group 4 & 25 & $0.301 \pm 0.216$ & \\
\hline
\end{tabular}




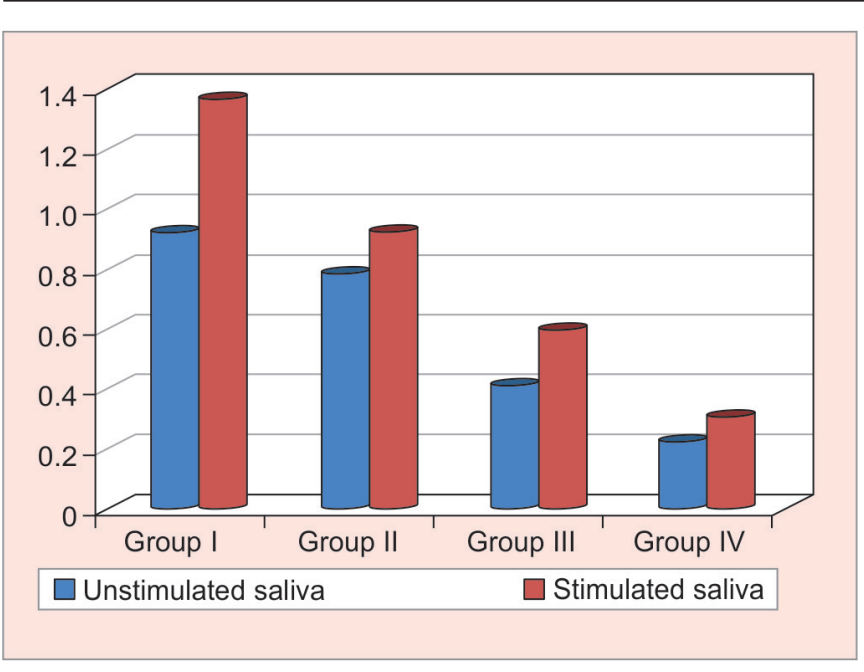

Graph 1: Salivary flow rate with chronic periodontitis patients

The data proved to be significant statistically with a $p$ value less than $0.05(p<0.05)$.

\section{DISCUSSION}

There are many studies which focus on the association of the periodontal disease with salivary pathogens. ${ }^{8,9}$ It is commonly known that the saliva harbors the periodontal pathogens, but the association of the periodontal pathogens with the salivary flow rate is unknown. The salivatory flow rate associated with the periodontal pathogens reflects the patient's periodontal condition. This phenomenon is studied extensively, the research suggesting the possible relationship between the salivary parameters with periodontitis and gingivitis is very little. ${ }^{3}$

The prevalence of the chronic periodontal disease involves persistence inflammation characterized by the formation of biofilms of gram-positive and gram-negative bacteria, leading to breaking the connective tissue attachment with the tooth structure further leading to alveolar bone destruction which causes mobility of the tooth and tooth loss. ${ }^{10}$

The present study result showed the significant correlation between the salivary flow rate with the chronic periodontal disease with a $p$ value less than 0.05 . This finding is compared with the studies conducted by Farsi et al., ${ }^{11}$ Marton et al. ${ }^{12}$ which showed the low resting salivary flow rate in patients with periodontal disease. The studies by Almstahl and Wikstrom, ${ }^{13}$ Hirotomi et al. ${ }^{14}$ showed no significant relation between periodontal disease with saliva. These finding turned out to be inconclusive to understand the complex role of saliva responding to the periodontal disease occurrence.

The study results from Shaila et al..$^{15}$ turned out to be completely different from the present study, which showed no correlation between salivary flow rate and periodontitis nor with healthy individuals. There was no usage of the standard protocol to collect the saliva, which might have led to irrelated results. In the study, $5 \mathrm{~mL}$ of saliva was collected without any restriction in the time duration, the flow rate was calculated with the different time duration which differed in each patient. To determine the qualitative composition of the saliva, $5 \mathrm{~mL}$ of saliva was collected. The selection criteria for periodontitis included based on the loss of attachment with a pocket depth of $\geq 5 \mathrm{~mm}$ at least at eight sites. This criterion might have affected the consideration of localized periodontal disease. There are chances of miss interpretation of reduced periodontium and gingivitis as periodontitis.

In the present study, the salivary flow rate was measured in two ways. The salivary gland's functional capacity was measured by stimulated salivary flow rate, which can also be used as a marker to detect the extent of glad dysfunction, whereas the amount of saliva which is constantly secreted into the oral cavity was measured by unstimulated salivary flow rate method. The decrease in the stimulated salivary flow rate showed to be significantly associated with the increase in the oral cavity anaerobic bacteria during chronic periodontitis condition as per the study conducted by Loesche et al. ${ }^{16}$

The salivary gland capacity is significantly affected by conditions other than periodontitis causing secretory dysfunction, but then there is no strong proof for clarification. The mechanism probably related is due to increase in the proinflammatory cytokine levels [tumor necrosis factor- $\beta$ (TNF- $\beta$ ), interleukin (IL-17), IL-2] during periodontitis which causes hyposalivation trigged by glandular dysfunction. ${ }^{17}$ The production of reactive oxygen species (ROS) is speeded up by the hyperresponsive polymorphoneutrophils (PMNs), which is activated by inflammatory cytokines. As that of physiological aging, the periodontitis leads to an increase in the buildup of oxidative stress causing structural changes. ${ }^{18}$ This study might be the first to show the periodontal disease effect on salivary glands and its flow like the best of our knowledge.

The inflammatory condition of the periodontal tissue is called periodontitis. The increase in the salivary flow rate can lead to an increase in the crevicular fluid which is an inflammatory exudate. As a defense mechanism, increased salivary flow rate delivers the immune cells along with inflammatory mediators to the infection site. There are many functions of saliva such as cleansing action, control of $\mathrm{pH}$, importantly antimicrobial activity. ${ }^{19,20}$ The oral cavity is protected from infectious microorganisms by the presence of neutrophils, antimicrobial peptides, thiocyanates and other molecules with antimicrobial properties in the whole saliva. Therefore, it can be expected to have an inflammatory response co-inside with the increase in the salivary flow rate as a reasonable body response. 


\section{CONCLUSION}

The present study proves the significant decrease in the stimulated and unstimulated salivary flow with severe progression of chronic periodontitis.

\section{REFERENCES}

1. Loe H. Periodontal disease. The sixth complication of diabetes mellitus. Diabetes Care1993;16:329-334.

2. Nunn M. Understanding the etiology of periodontitis: an overview of periodontal risk factors. Periodontology 2000, 2003;32:11-23.

3. Zulkarnain S, Azirrawani A. Association between Salivary Parameters and Periodontal Disease. International Medical Journal 2013;20(5):1-5.

4. Dodds MW, Johnson DA, Yeh CK. Health benefits of saliva: a review. J Dent 2005;33: 223-233.

5. Shimazaki $Y$ et al. Stimulated salivary flow rate and oral health status. Journal of Oral Science 2016:1-7.

6. Foglio-Bonda PL, Migliario M, Rocchetti V, Pattarino F, FoglioBonda A. Daily and annually variation of unstimulated whole saliva flow rate and $\mathrm{pH}$ and their relation with body profile in healthy young adults. Eur. Rev. Med. Pharmacol. Sci. 2013; 17(18):2538-2545.

7. Yamamoto K, Kurihara M, Matsusue Y, Imanishi M, Tsuyuki M, Kirita T. Whole saliva flow rate and body profile in healthy young adults. Arch. Oral Biol. 2009; 54(5):464-469.

8. Donna L, Anne D, Haffajee, Socransky S. Effects of periodontitis and smoking on the microbiota of oral mucous membranes and saliva in systemically healthy subjects. J Clin Periodontol 2003; 30(12):1031.

9. Larmas M. Saliva and dental caries: diagnostic tests for normal dental practice. Int Dent J 1992;42(4):199-208.

10. Abiodun O. Arigbede, B. OsagbemiroBabatope, and M. Kolude Bamidele Periodontitis and systemic diseases: A literature review. J Indian Soc Periodontol. 2012 Oct-Dec; 16(4): 487-491.
11. Farsi N, Al Amoudi N, Farsi J, Bokhary S, Sonbul H. Periodontal health and its relationship with salivary factors among different age groups in a Saudi population. Oral Health Prev Dent2008;6:147-154.

12. Marton K, Madlena M, Banoczy J, et al. Unstimulated whole saliva flow rate in relation to sicca symptoms in Hungary. Oral Dis2008;14:472-477.

13. Almstahl A, Wikstrom M. Oral microflora in subjects with reduced salivary secretion. J Dent Res 1999;78(8):1410-1416.

14. Hirotomi T, Yoshihara A, Ogawa H, Ito K, Igarashi A, Miyazaki H. Salivary spinability and periodontal disease progression in an elderly population. Arch Oral Biol, 2008;53: 1071-1076.

15. Shaila M, Pai GP, Shetty P. Salivary protein concentration, flow rate, buffer capacity and $\mathrm{pH}$ estimation: A comparative study among young and elderly subjects, both normal and with gingivitis and periodontitis. Journal of Indian Society of Periodontology 2013;17(1):42-46.

16. Loesche WJ, Schork A, Terpenning MS, Chen YM, Stoll J. Factors which influence levels of selected organisms in saliva of older individuals. J Clin Microbiol. 1995 Oct;33(10): 2550-2557.

17. Bhattarai KR, Junjappa R, Handigund M, Kim HR, Chae HJ. The imprint of salivarysecretion in autoimmunedisorders and related pathological conditions. Autoimmun Rev. 2018 Apr;17(4):376-390.

18. Yoshitaka Yamauchi, Tomonori Matsuno, Kazuhiko Omata, and Tazuko Satoh.Relationship between hyposalivation and oxidative stress in aging mice.J Clin BiochemNutr. 2017 Jul; 61(1): 40-46.

19. Hamada T NT, Kimura T, Arisawa K, Yoneda K, Yamamoto T, Osaki T. Treatment of xerostomia with the bile secretionstimulant drug anethole trithione: A clinical trial. Am J Med Sci 1999;318:6.

20. McDonald E, Marino C. Dry mouth: diagnosing and treating its multiple causes. Geriatrics. 1991 Mar;46(3):61-63. 